Dom. Cien., ISSN: 2477-8818

Vol. 4, núm. 1, enero, 2018, pp. 499-510

Las TICs en las empresas: evolución de la tecnología y cambio estructural en las organizaciones

\title{
Las TICs en las empresas: evolución de la tecnología y cambio estructural en las organizaciones
}

\author{
ICTs in companies: evolution of technology and structural change in \\ organizations
}

\section{TIC nas empresas: evolução da tecnologia e mudanças estruturais nas organizações}

\author{
Galo E. Cano-Pita ${ }^{\mathrm{I}}$ \\ galo-cano@hotmail.com \\ Mariana J. García-Mendoza II \\ angellos_69@hotmail.com
}

Recibido: 10 de septiembre de 2017 * Corregido: 10 de noviembre de 2017 * Aceptado: 27 de diciembre de 2017

I Magister en Marketing, Magister en Docencia Universitaria e Investigación Educativa, Licenciado en Mercadotecnia, Ingeniero en Marketing y Finanzas, Ingeniero en Marketing y Finanzas, Docente de la Universidad San Gregorio de Portoviejo, Portoviejo, Ecuador.

II Magister en Gerencia Educativa, Profesora de Segunda Enseñanza en la Especialización de Asignaturas Secretariales, Licenciada en Ciencias de la Educación Especialidad Secretariado Bilingüe Computarizado, Unidad Educativa del Milenio "Carlos María Castro" Parroquia Santa Rita, Chone, Ecuador 


\section{Resumen}

El propósito del presente artículo, es analizar la importancia del cambio estructural de las organizaciones empresariales, de cara a la evolución de la tecnología, especialmente la implementación y uso de las Tic's en las empresas y los cambios en la estructura de las mismas, como medio para una mayor competitividad a largo plazo y mejor rendimiento general. La metodología utilizada ha sido de carácter documental, realizándose una revisión bibliográfica y un análisis de la información, se trata de un estudio descriptivo que busca especificar las propiedades, las características y los aspectos importantes del uso de la tecnología en las empresas y el cambio en la estructura de las mismas que conlleva. En tal sentido, se concluyó que existe una relación directa entre la evolución de la tecnología y el cambio estructural en las organizaciones, producto de la adopción por parte de las mismas, de nuevas tecnologías de información y comunicación, adoptadas inicialmente como herramientas para afrontar nuevas realidades del entorno, lo cual produjo posteriormente en ellas cambios profundos en su diseño estructural, cultura y clima organizacional. Por otra parte, se estableció la importancia de los sistemas de información, en cuanto a que permiten la difusión, no sólo de información para la toma de decisiones, sino de los conocimientos, las capacidades intelectuales de los seres humanos, los valores culturales, las habilidades, la experiencia, inclusive los modelos mentales. Dichos sistemas podrán convertirse en una potente herramienta que permita crear valor a las empresas y su estructura organizativa.

Palabras clave: tic's; tecnología; empresa; estructura; importancia.

\section{Abstract}

The purpose of this article is to analyze the importance of the structural change of business organizations, in the face of the evolution of technology, especially the implementation and use of Tic's in companies and the changes in their structure, as a means of for greater long-term competitiveness and better overall performance. The methodology used has been of a documentary nature, carrying out a bibliographic review and an analysis of the information, it is a descriptive study that seeks to specify the properties, characteristics and important aspects of the use of technology in companies and the change in the structure of them that entails. In this regard, it was concluded that there is a direct relationship between the evolution of technology and structural change in organizations, as a result of the adoption by them of new information and communication 
technologies, initially adopted as tools to face new realities of the environment, which subsequently produced in them profound changes in its structural design, culture and organizational climate. On the other hand, the importance of information systems was established, in that they allow the dissemination, not only of information for decision-making, but also of knowledge, the intellectual capacities of human beings, cultural values, skills, experience, including mental models. These systems can become a powerful tool to create value for companies and their organizational structure. Keywords: tic's; technology; company; structure; importance.

\section{Resumo}

O objetivo deste artigo é analisar a importância da mudança estrutural das organizações empresariais, diante da evolução da tecnologia, especialmente a implementação e uso da Tic em empresas e as mudanças em sua estrutura, como meio de para maior competitividade a longo prazo e melhor desempenho geral. A metodologia utilizada tem sido de natureza documental, realizando uma revisão bibliográfica e uma análise da informação, é um estudo descritivo que procura especificar as propriedades, características e aspectos importantes do uso da tecnologia nas empresas e a mudança de a estrutura deles que implica. A este respeito, concluiu-se que existe uma relação direta entre a evolução da tecnologia e as mudanças estruturais nas organizações, como resultado da adoção por elas de novas tecnologias de informação e comunicação, inicialmente adotadas como ferramentas para enfrentar novas realidades do meio ambiente, que posteriormente produziram mudanças profundas em seu design estrutural, cultura e clima organizacional. Por outro lado, a importância dos sistemas de informação foi estabelecida, na medida em que permitem a disseminação, não apenas de informação para a tomada de decisões, mas também do conhecimento, das capacidades intelectuais dos seres humanos, dos valores culturais, habilidades, experiência, incluindo modelos mentais. Esses sistemas podem se tornar uma ferramenta poderosa para criar valor para empresas e sua estrutura organizacional.

Palavras chave: tic's; tecnologia; empresa; estrutura; importancia.

\section{Introducción}

En la actualidad existen numerosos cambios en los mercados, competencias, organizaciones, tecnologías, sociedades y culturas, entre otros, razón por la cual se considera poco pertinente seguir maniobrando bajo el mismo enfoque tradicional. Para lograr ser competitivo dentro de este entorno 
tan cargado de dinamismo y turbulencia, es indispensable buscar ventajas competitivas y por ende un desarrollo económico a largo plazo, así como también desarrollar capacidad para producir, circular y utilizar correctamente la información, la comunicación y el conocimiento, por cuanto ellos constituyen la materia prima de esta nueva sociedad.

Desde tal perspectiva, (Mujica, 2000), considera que el avance tecnológico de la informática, la computación, y las telecomunicaciones, incorporaron en las organizaciones un enfoque diferente al habitual para acceder al conocimiento, flexibilidad, interactividad, economía, rapidez, independencia, comunicación y desarrollo.

Es un novedoso prototipo impulsado por la plataforma tecnológica, como lo refieren (Negroponte, 1996) y (Gates, 2000), se trata de la integración de las redes de teléfonos, televisión, cable, radio, computación e internet, las cuales logran la circulación de toda la información y comunicación, fundamental para satisfacer las necesidades de las organizaciones y garantizarles un futuro sustentable.

Las TIC, según (Gil, 2002), constituyen un conjunto de aplicaciones, sistemas, herramientas, técnicas y metodologías asociadas a la digitalización de señales analógicas, sonidos, textos e imágenes, manejables en tiempo real. Por su parte, (Ochoa \& Cordero, 2002), establecen que son un conjunto de procesos y productos derivados de las nuevas herramientas (hardware y software), soportes y canales de comunicación, relacionados con el almacenamiento, procesamiento y la transmisión digitalizada de la información. Asimismo, (Thompson \& Strickland, 2004) definen las tecnologías de información y comunicación, como aquellos dispositivos, herramientas, equipos y componentes electrónicos, capaces de manipular información que soportan el desarrollo y crecimiento económico de cualquier organización.

Esta evolución se ha visto impulsada por las nuevas tecnologías de información, que han permitido incluso a los especialistas de la economía ver como sus hipótesis teóricas se acercan a la realidad al incrementar la información disponible, información que confirma como las empresas se ven en la necesidad imperiosa de reajustar sus estructuras organizacionales, pasando de modelos tradicionales jerárquicos orientados al mando vertical propios de la sociedad industrial, a estructuras que tienden cada vez más a la línea de mando horizontal por su eficiencia en el manejo de la información, siendo 
la tecnología la herramienta fundamental y la información el insumo necesario propios de la sociedad del conocimiento. (Drucker, 1993).

Así como el teléfono como tecnología de punta, en una época marco cambios drásticos dentro de la organización, donde el gerente podía impartir órdenes a distancia tanto a niveles jerárquicos dentro empresa como a unidades geográficamente distantes, hecho este que produjo cambios contundentes en sus estructuras; hoy en día este fenómeno se repite con la incorporación de las TIC (computadoras, las redes de comunicación los sistemas de información etc.). Por otra parte, el concepto de cambio organizativo se está identificando y relacionando cada vez más con el avance de la economía del conocimiento, pues son las empresas más innovadoras y creadoras de conocimiento las que están permanentemente evolucionando, y más allá de los aspectos mencionados, el debate sobre las nuevas estructuras debe centrarse en la relación entre la creación de capital intelectual y el diseño estructural en la que el concepto clave para las organizaciones es la información, de allí que la estructura debe centrarse en facilitar eficazmente la obtención, procesamiento y aprovechamiento de la información para contribuir a la calidad y el desempeño. En este orden de ideas (Benjamín \& Blunt, 1992) afirman en sus estudios que el surgimiento de las nuevas configuraciones organizativas han coincidido y han sido coadyuvadas por el desarrollo de las TIC, a las que se les suele atribuir una función fundamentalmente flexibilizadora y un carácter dinamizador de la organización, quedando en su investigación planteada la necesidad de contrastar en qué medida y en qué dirección las TIC afectan los rasgos del diseño organizativo y cómo se ajustan entre sí ambos elementos, para dar respuesta a la cuestión crucial del cambio organizativo. En relación a esta interrogante y tomando como base el trabajo de los autores (Benjamín \& Blunt, 1992), los investigadores (Laud \& Thies, 1997) concluyen en su trabajo que efectivamente las TIC no sólo contribuyen al cambio, sino que además lo potencian, dándole flexibilidad a la empresa, permitiendo agilizar cambios tanto estructurales como culturales, distribuyendo la información a toda la organización de manera rápida y eficiente; por otro lado, concluyen también en su trabajo que las TIC son capaces de proporcionar sistemas de control y de planificación más integral, que favorecen un análisis global de los datos por parte de una persona en particular o la mayoría de actores que conforman la organización, en definitiva, se trata de proveer la herramienta necesaria para promover la toma de decisiones a cualquier área de la organización. Según (Phillips, 1995) cuando se hace referencia a evolución, de inmediato se tiene que pensar en "cambios", entonces es allí donde hay que fijar la concentración para identificar de manera clara y precisa qué 
aspectos de la organización cómo y cuándo se verán afectados por los mismos, tales como: la desaparición de algunas rutinas organizativas y con ellas parte del conocimiento acumulado en los procesos asociados a las mismas, cambios en las estructuras tanto administrativas como de gerencia y control, cambios que se reflejaran tanto en la cultura como el clima organizacional, muchos de ellos producto del rechazo por parte del capital humano a explorar nuevas situaciones, que le generen incertidumbre, nuevas formas de gestionar la información (recibir, distribuir, almacenar, transformar y transmitir), aprendizaje y adecuación al uso de nuevas tecnologías.

Las TICs son esenciales para mejorar la productividad de las empresas, la calidad, el control y facilitar la comunicación, entre otros beneficios, aunque su aplicación debe llevarse a cabo de forma inteligente.

El mero hecho de introducir tecnología en los procesos empresariales no es garantía de gozar de estas ventajas. Para que la implantación de nueva tecnología produzca efectos positivos, hay que cumplir varios requisitos: tener un conocimiento profundo de los procesos de la empresa, planificar detalladamente las necesidades de tecnología de la información e incorporar los sistemas tecnológicos paulatinamente, empezando por los más básicos.

Antes de añadir un componente tecnológico, hay que conocer bien la organización y/o empresa. Se ha descubierto que el $90 \%$ de las veces, el fracaso no es debido al software ni a los sistemas, sino al hecho de que la gente no tiene suficientes conocimientos sobre su propia empresa o sus procesos empresariales.

Otro aspecto importante a considerar es que las empresas que tienen una gran capacidad de beneficiarse de la tecnología, son organizaciones que, antes de añadir un componente tecnológico, describen detalladamente cuál será la repercusión para su empresa.

Las tecnologías de la información y la comunicación, han transformado nuestra manera de trabajar y gestionar recursos. Las TIC son un elemento clave para hacer que nuestro trabajo sea más productivo: agilizando las comunicaciones, sustentando el trabajo en equipo, gestionando las existencias, realizando análisis financieros, y promocionando nuestros productos en el mercado. 
El buen uso de las TIC permite a las empresas producir más cantidad, más rápido, de mejor calidad, y en menos tiempo y le ayudan a la competitividad.

\section{Materiales y métodos}

La metodología utilizada ha sido de carácter documental, realizándose una revisión bibliográfica y un análisis de la información.

Según (Morales, 2003) la investigación documental tiene la particularidad de utilizar como una fuente primaria de insumos, mas no la única y exclusiva, el documento escrito en sus diferentes formas: documentos impresos, electrónicos y audiovisuales. La investigación documental es un procedimiento científico, un proceso sistemático de indagación, recolección, organización, análisis e interpretación de información o datos en torno a un determinado tema. Al igual que otros tipos de investigación, éste es conducente a la construcción de conocimientos.

Ha de entenderse que los estudios descriptivos "buscan especificar las propiedades, las características y los aspectos importantes del fenómeno que se somete a análisis. (Gómez, 2006).

Así mismo, de la literatura antes referida, sirve de apoyo para fundamentar éste trabajo investigativo, puesto que en dicha fuente se explica que los estudios descriptivos, se enfocan en recabar datos que muestren cómo se manifiesta un hecho, contexto, evento, fenómeno o situación. De la misma manera, el autor refiere que la descripción puede ser más o menos profunda, y en cualquiera de los casos se basa en la recolección de datos sobre éste y su contexto, en relación específica con las investigaciones de variable cualitativa, como es el presente caso.

\section{Resultados}

Vemos entonces como la variable tecnología siempre está presente en cada etapa del desarrollo de la estructura empresarial, por lo cual se podría concluir una relación muy estrecha entre evolución y tecnología. Con base a los estudios de algunos investigadores del área revisados en la presente investigación, se puede inferir de manera concluyente la relación directa que existe entre la evolución y cambio estructural en las organizaciones, producto de la adopción por parte de las mismas de nuevas tecnologías de información y comunicación, adoptadas inicialmente como herramientas para afrontar 
nuevas realidades del entorno, lo cual produjo posteriormente en ellas cambios profundos en su diseño estructural, cultura y clima organizacional.

Es importante destacar que en ambientes tan complejos como los que deben enfrentar hoy en día las organizaciones, sólo aquellos que utilicen todos los medios a su alcance, y aprendan a aprovechar las oportunidades del mercado visualizando siempre las amenazas, podrán lograr el objetivo de ser exitosas.

La gerencia de las organizaciones del futuro debe administrar de manera enérgica el insumo tecnológico, como lo plantea (Cuesta, 2000), no se pueden adoptar todos los nuevos avances tecnológicos, sin antes considerar los posibles efectos colaterales que se producirán en el entorno político, económico, social, educativo, cultural y ambiental, así como también en los diferentes niveles geográficos; local, nacional e internacional.

La innovación tecnológica aparece como una condición esencial para la expansión de la sociedad, de forma que el desarrollo de Know - how y el cambio tecnológico vienen a ser impulsores del crecimiento sostenido (Gaynor, 1999). Ello es en parte resultado del carácter cada vez más interdisciplinado de los avances tecnológicos, como derivación de la fusión y afinidad entre las áreas tecnológicas atractivas, ofrecidas por los medios de comunicación e información, los cuales se han convertido en la era de la información y el conocimiento, en el ápice central de toda organización que avizora el éxito la rentabilidad y la competitividad a largo plazo.

El cambio tecnológico, sostiene (Porter, 2000), que no es importante por sí mismo, pero es fundamental si afecta la ventaja competitiva y la estructura del sector donde opera. No todo cambio relacionado con este enfoque es estratégicamente benéfico, puede empeorar en muchas ocasiones la posición competitiva de la organización y lo atractivo de la fracción involucrada, lo que sin duda amerita una planificación, organización, dirección y control de la gestión tecnológica, con el firme propósito de evaluar la inclusión e impacto de las tecnologías en el marco empresarial.

En definitiva, las tecnologías han hecho parte importante en la vida de todo ser humano. (Castells, 1998), sostiene que las tecnologías dominantes de una sociedad producen consecuencias sociales, que determinan la vida de las personas, por cuanto las innovaciones tecnológicas obligan a los seres humanos a adoptar una nueva forma de vida con valores nuevos. Esto implica que sean modificadas 
o eliminadas muchas capacidades humanas, pero se extienden otras, gracias a esto se puede observar cómo se han movido las tecnologías en el tiempo y su influencia sobre la sociedad en una especie de relación simbiótica.

La búsqueda constante del hombre por satisfacer cada día más sus necesidades de comunicación, ha sido impulso para lograr la instauración en el mundo de instrumentos cada día más poderosos y veloces en el proceso comunicativo. Sólo basta una retrospectiva, según (Pablos, 2001), para definir como el ser humano ha logrado evolucionar sus formas de integración y diálogo pasando por la invención del alfabeto y el papel, hasta los actuales satélites y telecomunicaciones, y donde prácticamente todos han sido posibles gracias a la tecnología, quien a su vez ha sido el instrumento cuyo adelanto ha determinado el avance de la humanidad.

La competitividad, es una condición para enfrentar con éxito el reto de penetrar los mercados internacionales, (Hernández, 1999), sostiene que también es factor clave para liderizar los mercados internos, de allí que ser competitivo es llegar al público, y a los clientes con productos y servicios que satisfagan sus requerimientos y expectativas.

En este sentido, (Licha, 1998), expone, que para buscar la competitividad se pueden mencionar algunas herramientas como: acertado manejo de la información, existencia de talleres cónsonos con las realidades de las plantas, existencia de centros de documentación, de manuales de procedimientos, de planos, de equipos y de procesos, entre otros, que permitan a las organizaciones acomodarse en la producción, de acuerdo con los patrones internacionales de calidad y precios, lo cual necesariamente lleva a contar con capacidad tecnológica.

De lo anterior se desprende, que el factor tecnológico es determinante para la competitividad en el mercado internacional, de allí que, para lograr el crecimiento económico, los países se basen en la capacidad de innovación que éstos posean.

\section{Conclusiones}

Con base a lo anteriormente descrito, se puede concluir que la capacidad de gestión de las Tecnologías de Información y Comunicación (TIC), proporcionan una ventaja competitiva, y no tanto las capacidades técnicas o la infraestructura. Es fundamental el análisis de los factores afectados por la 
implementación de un nuevo producto tecnológico, para saber cuál ha de ser la estrategia que debe adoptar la organización frente al cambio, pero ciertamente es imperioso que la organización empresarial vaya de la mano con la evolución de la sociedad, los avances tecnológicos y la globalización.

Las TIC deben impulsar un cambio organizacional total y no sólo un cambio técnico. Es vital que el personal de las empresas protagonice el cambio y venzan su natural resistencia. Deben comprender que el puesto de trabajo se transforma, pero no se desaparece, eliminando lo accesorio y alienante en aras de tareas más creativas y motivadoras. Es esencial para las empresas no quedarse atrás y acometer una renovación desde sus TIC que les permita diseñar los sistemas de información más acorde con sus planteamientos estratégicos para disponer y suministrar de la información adecuada en el momento preciso. Esto lleva consigo, una reestructuración organizativa y una reingeniería de procesos que puede asustar, inicialmente, a las empresas con menos medios o capacidad de evolución pero que les sitúa ante una disyuntiva clásica: renovarse o morir.

Las empresas se enfrentan a un reto que supera la simple renovación tecnológica. Es un cambio social, económico y cultural que deben empezar a planificar hoy. El conocimiento y su gestión se están convirtiendo en un recurso y habilidad que tienen la capacidad de crear valor para aquellas empresas que lo utilicen en todas sus extensiones. Los sistemas y las tecnologías de la información no son ajenos a este cambio. Así pues, los sistemas de información que permitan la difusión, no sólo de información para la toma de decisiones, sino de los conocimientos, las capacidades intelectuales de los seres humanos, los valores culturales, las habilidades, la experiencia, inclusive los modelos mentales, pueden convertirse en una potente herramienta que permita ayudar a los seres humanos en todas sus actividades y al mismo tiempo crear valor a la empresa.

La tecnología por sí sola no beneficia a la organización, es necesario que ésta se incorpore a las actividades cotidianas mediante la formación de los empleados. Asimismo, la tecnología aislada, no cambia los procesos de producción o comercialización, si no está respaldada de planes de negocios que controlen y definan los objetivos de su uso. Para extraer de las TIC todo su potencial, su implantación y desarrollo, éstas han de contemplarse en el contexto de una estrategia tecnológica sostenible en el tiempo. 


\section{Referencias Bibliográficas}

Benjamín, \& Blunt. (1992). Critical IT Issues: The Next Ten Years. Sloan Management Review, 33(4), 7-19

Castells, M. (1998). La era de la información. España: Mc Graw Hill

Cuesta, S. (2000). La empresa virtual. España: Mc Graw Hill

Drucker, P. (1993). Administración para el futuro. España: Parragón

Gates, B. (2000). Los negocios en la era digital. España: Plaza \& James Editores, S.A

Gaynor, G. (1999). Manual de gestión en tecnología. Una estrategia para la competitividad de las empresas. Colombia: Mc Graw Hill

Gil, E. (2002). http:www.voc.edu/web/esplart/gil0902/htm. Recuperado el 02 de mayo 2017, de http:www.voc.edu/web/esplart/gil0902/htm

Gómez, M. (2006). Introducción a la metodología de la investigación científica. Argentina: brujas

Hernández, E. (1999). Competitividad y estrategias empresariales. Acta científica Venezolana

Laud, \& Thies. (1997). Great Expectations: Structuring IT Organizations That Really Deliver. Business Horizons, 40(4), 25-36

Licha, I. (1998). Indicadores de gestión de la investigación y el desarrollo tecnológico. Red iberoamericana de ciencia y tecnología. España: nueva sociedad

Morales, O. (2003). Fundamentos de la investigación documental y la monografía. Facultad de Odontología de la Universidad de Los Andes. Manual para la elaboración y presentación de la monografía. Mérida, Venezuela

Mujica, M. (2000). Nuevas estrategias para gerenciar. Una visión epistemológica. UNESR, 61-76

Negroponte, N. (1996). Ser digital. España: Ediciones B.S.A 
Ochoa, \& Cordero.

(2002).

http://www.ruv.itesm.mx/especiales/citela/documentos/material/módulos/módulos2/contenidoii.htm.

$\begin{array}{llllll}\text { Recuperado } & \text { el } & 02 & \text { de } & \text { mayo }\end{array}$

http://www.ruv.itesm.mx/especiales/citela/documentos/material/módulos/módulos2/contenidoii.htm

Pablos, J. d. (2001). La red es nuestra. España: Ediciones Paidos

Phillips, N. (1995). Motivating for change. Inglaterra: pitman

Porter, H. (2000). Ventaja competitiva. Creación y sostenimiento de un desempeño superior. México: Continental

Thompson, \& Strickland. (2004). Administración estratégica. México: Mac Graw Hill 